\title{
Electron-acoustic Phonon Interaction in AlAs/GaAlAs Resonance Tunneling Nanostructures
}

\author{
I.V. Boyko, M.R. Petryk
}

Ternopil Ivan Puluj National Technical University, 56, Ruska St., 46001 Ternopil, Ukraine

(Received 23 June 2020; revised manuscript received 15 December 2020; published online 25 December 2020)

\begin{abstract}
In paper, using exact solutions of the stationary Schrödinger equation and the equation of motion for an elastic semiconductor medium, using the secondary quantization formalism, the theory of interaction of electrons with acoustic phonons in a multilayer arsenide-based AlAs/GaAlAs resonant tunneling structure is developed. Using the Matsubara Green's functions and the Dyson equation, expressions, which describe the temperature energy shifts of electronic levels in the nanostructure and their decay rates, are established. Direct calculations of the quantities characterizing the interaction of electrons with acoustic phonons are performed on the basis of physical and geometric parameters of a typical nanostructure, and their dependences on the geometric design of the total potential well of the nanosystem at various temperatures are studied. It is shown that the influence of acoustic phonons leads to the decrease in the quantum electronic transitions frequency in the studied nanostructure, and this effect becomes more noticeable with increasing temperature. It has been established that the absolute values of the electronic stationary states temperature shifts decreases with the increase in the electronic stationary level number. Also, an increase in the temperature entails an increase in the electronic states decay rates that is a dissipation effect directly affecting electronic processes in nanostructures.
\end{abstract}

Keywords: Acoustic phonon, Electron-phonon interaction, Dyson equation, Energy shift, Decay rate.

DOI: 10.21272/jnep.12(6).06030

PACS numbers: 63.22.Np, 63.20.kd

\section{INTRODUCTION}

The development of modern nanoscale devices that can operate in the terahertz and infrared ranges of electromagnetic waves, in particular, quantum cascade lasers (QCLs) and detectors (QCDs) [1-4], is a main part of semiconductor physics and technology. It leads to significant scientific and technical interest in multilayer semiconductor resonant tunneling structures (RTS) - plane nanosystems, which are precision active elements of the cascades for mentioned devices.

Considerable attention of theoretical physicists working in this direction extends to the study of the spectra and interaction of quasiparticles in multilayer RTS.

The interaction of electrons with optical phonons in such nanosystems is studied quite well $[5,6]$. However, in the case of acoustic phonons, this direction remains poorly studied. The spectra and components of the displacement field for acoustic phonons were mainly studied for single-well nanosystems [7-9] placed in an external non-stressed medium, which simplified the application of boundary conditions for the components of the displacement field and the stress tensor.

In the same simplified model, the interaction of electrons with acoustic phonons in the trivial case of temperature $(T=0 \mathrm{~K})$ was also studied. For a more realistic model of multilayer RTS, the problem of acoustic phonons investigation was recently solved for both homogeneous arsenide-based AlAs/AlGaAs [10] and anisotropic AlN/GaN [11] nanosystems. However, interaction with acoustic phonons in multilayer nanostructures still remains an unsolved problem.

In the present paper, a quantum mechanical theory of the interaction of electrons with acoustic phonons in the multilayer RTS is developed. The spectra of electrons and acoustic phonons were calculated. Direct calculations performed for the double-well active band of the QCL or QCD show, that the developed theory de- scribes well the shifts of the electronic spectrum and electronic states decay rates due to the electron-phonon interaction in a wide temperature range.
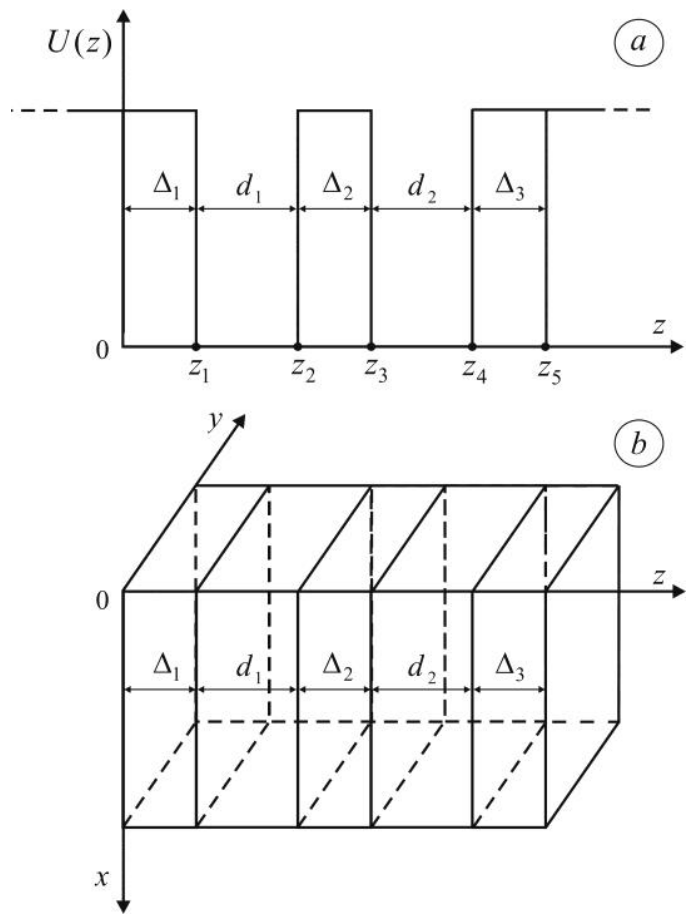

Fig. 1-Geometric (a) and energy (b) schemes of closed threebarrier RTS

\section{THEORY OF STATIONARY ELECTRONIC STATES IN A MULTILAYER NANOSTRUCTURE}

First, we will study stationary electronic states in a three-barrier RTS, the energy scheme of which is shown in Fig. 1a. 
Taking into account the notation given in Fig. 1a, the potential energy of an electron $U(z)$ and its effective mass $m(z)$ in the studied nanosystem can be presented using unit Heaviside function $\theta(z)$ as follows:

$$
\begin{gathered}
U(z)=U_{0}\left\{\theta(-z)+\theta\left(z-z_{5}\right)+\right. \\
+\sum_{p=0}^{2}\left[\left(\theta\left(z-z_{2 p}\right)-\theta\left(z-z_{2 p+1}\right)\right]\right\} \\
m(z)=m_{w} \sum_{p=1}^{3}\left(\theta\left(z-z_{2 p-1}\right)-\theta\left(z-z_{2 p}\right)\right)+ \\
+m_{b}\left\{\theta(-z)+\sum_{p=0}^{2}\left[\theta\left(z-z_{2 p}\right)-\theta\left(z-z_{2 p+1}\right)\right]+\theta\left(z-z_{5}\right)\right\},
\end{gathered}
$$

where, $m_{w}$ and $m_{b}$ are the effective masses of an electron, respectively, in semiconductor layers for GaAlAs potential wells and AlAs potential barriers, $U_{0}=0.9\left(E_{g}(\mathrm{AlAs})-E_{g}(\mathrm{AlGaAs})\right)$ is the value of the potential barrier. Bandgap $E_{g}$ for arsenide $\mathrm{Ga}_{1-x} \mathrm{Al}_{x} \mathrm{As}$ semiconductor at temperature $T$ is calculated due to Varshni ratio

$$
E_{g}(x, T)=E_{g}(x, 0)+\alpha(x) / T^{2}(\beta(x)+T),
$$

where bandgap at $T=0 \mathrm{~K}$ and Varshni parameters depending on the value of $x$ are as follows [12]

$$
E_{g}(x, T)=E_{g}(x, 0)+\alpha(x) / T^{2}(\beta(x)+T),
$$

$$
\begin{aligned}
& E_{g}(x, 0)=x E_{g}(\mathrm{AlAs})+(1-x) E_{g}(\mathrm{GaAs}) \\
& E_{g}(\mathrm{AlAs})=2239(\mathrm{meV}) ; E_{g}(\mathrm{GaAs})=1521(\mathrm{meV}) \\
& \alpha(x)=(6.00 x+5.58(1-x)) \cdot 10^{-4}(\mathrm{eV} / \mathrm{K}) \\
& \beta(x)=408 x+220(1-x)(\mathrm{K}) .
\end{aligned}
$$

For stationary electronic states, that is for $E \leq U_{0}$, we represent the wave function of an electron in the nanosystem as follows

$$
\Psi(E, x, y, z)=\Psi_{E \bar{k}}(E, z)=\frac{1}{\sqrt{l_{x} l_{y}}} e^{i \bar{k} \bar{r}} \Psi(E, z),
$$

where $\bar{k}$ is the electron quasi-momentum and $\bar{r}$ is the vector in the plane corresponding to the cross-section of the nanosystem by the $x O y$ plane, $l_{x}, l_{y}$ are the geometrical parameters of this cross-section.

Thus, taking into account expression (6), the stationary spectrum for an electron and its wave functions it is necessary to find solutions for the stationary Schrödinger equation

$$
\left[-\frac{\hbar^{2}}{2} \frac{\partial}{\partial z}\left(\frac{1}{m(z)}\right) \frac{\partial}{\partial z}+U(z)\right] \Psi(E, z)=E \Psi(E, z) .
$$

Moreover, the total energy of an electron is expressed as the sum of the energies of the longitudinal and transverse movements:

$$
E_{n \bar{k}}=E_{\|}+E_{\perp}=E_{n}+\frac{\hbar^{2} k^{2}}{2 \bar{m}_{e f f}},
$$

where the effective electron mass averaged over the contributions of all layers of the nanosystem is determined as [5]

$$
\bar{m}_{e f f}=\int_{-\infty}^{+\infty}\left[\left|\Psi\left(E_{n}, z\right)\right| / m(z)\right] d z .
$$

The solutions of the Schrödinger equation taking into account (1) and (2) are

$$
\begin{aligned}
& \Psi(E, z)=\Psi^{(0)}(E, z) \theta(-z)+\Psi^{(6)}(E, z) e^{-\chi z} \theta\left(z-z_{5}\right)+ \\
& +\sum_{p=1}^{5} \Psi^{(p)}(E, z)\left[\theta\left(z-z_{p-1}\right)-\theta\left(z-z_{p}\right)\right]= \\
& =A^{(0)} e^{\chi z} \theta(-z)+B^{(6)} e^{-\chi z} \theta\left(z-z_{5}\right)+ \\
& +\sum_{p=1}^{5}\left(A^{(p)} e^{i K\left(z-z_{p-1}\right)}+B^{(p)} e^{-i K\left(z-z_{p-1}\right)}\right)\left[\theta\left(z-z_{p-1}\right)-\theta\left(z-z_{p}\right)\right] .
\end{aligned}
$$

where the following notation is introduced:

$$
K=\sqrt{2 m_{w} E} / \hbar ; \chi=\sqrt{2 m_{b}\left(U_{0}-E\right)} .
$$

In relation (10), it is taken into account that $B^{(0)}=A^{(6)}=0$, since the condition is fulfilled

$$
\underset{z \rightarrow \pm \infty}{\Psi(z) \rightarrow 0}
$$

Now, using the boundary conditions for the wave function (8)

$$
\left\{\begin{array}{l}
\left.\Psi^{(p)}(E, z)\right|_{z \rightarrow z_{p}-0}=\left.\Psi^{(p+1)}(E, z)\right|_{z \rightarrow z_{p}+0} ; \\
\left.\frac{1}{m(z)} \frac{d \Psi^{(p)}(E, z)}{d z}\right|_{z \rightarrow z_{p}-0}=\left.\frac{1}{m(z)} \frac{d \Psi^{(p+1)}(E, z)}{d z}\right|_{z \rightarrow z_{p}+0} .
\end{array}\right.
$$

the dispersion equation is obtained, which determines the stationary spectrum $E_{n}$ of an electron in the nanostructure. In addition, from relation (10) and conditions for normalizing the wave function

$$
\int_{-\infty}^{+\infty} \Psi_{E \bar{k}}\left(E_{n}, z\right) \Psi_{E^{\prime} \bar{k}^{\prime}}^{*}\left(E_{n^{\prime}}, z\right) d z=\delta_{n n^{\prime}} \delta_{\overline{k \bar{k}^{\prime}}}
$$

all coefficients $A^{(p)}, B^{(p)}$ are uniquely determined, and therefore the wave function $\Psi\left(E_{n}, z\right)$.

We now replace the wave functions with operators in the following form:

$$
\hat{\Psi}_{n \bar{k}}=\sum_{n \bar{k}} \Psi_{n \bar{k}}(\bar{r}, z) \hat{a}_{n \bar{k}},
$$

then we get the free electron Hamiltonian as follows

$$
\hat{H}_{e}=\sum_{n \bar{k}} E_{n \bar{k}} \hat{a}_{n \bar{k}}^{+} \hat{a}_{n \bar{k}}
$$

where $\hat{a}_{n \bar{k}}^{+}$and $\hat{a}_{n \bar{k}}$ are the fermionic creation and annihilation operators for the electronic stationary states.

\section{ACOUSTIC PHONON MODES IN PLANE ARSENIDE-BASED RESONANT TUNNELING STRUCTURES}

The components of the elastic displacement field associated with acoustic phonons and the modes of these 
phonons are obtained by the solutions of the equation of motion for a multilayer $\mathrm{AlAs} / \mathrm{GaAs}$ semiconductor layered medium:

$$
\begin{aligned}
& \rho(z) \frac{\partial^{2} \bar{u}(x, y, z)}{\partial t^{2}}=\frac{\partial \sigma_{i j}(x, y, z)}{\partial x_{j}}, j=1,2,3 ; \\
& x_{1}=x ; x_{2}=y, x_{3}=z .
\end{aligned}
$$

where the stress tensor has a form

$$
\sigma_{i j}(x, y, z)=C_{i j l m}(z) u_{l m}(x, y, z), l, m=(1 ; 2 ; 3)
$$

and the strain tensor is as follows

$$
u_{l m}(x, y, z)=\frac{1}{2}\left(\frac{\partial u_{l}(x, y, z)}{\partial x_{m}}+\frac{\partial u_{m}(x, y, z)}{\partial x_{l}}\right) .
$$

In relations (17) and (18), respectively, the RTS density and elastic constants, depending on the $z$ coordinate and notation in Fig. 1b, are in next the form:

$$
\begin{gathered}
\rho(z)=\sum_{p=1}^{6} \rho^{(p)}\left[\theta\left(z-z^{(p-1)}\right)-\theta\left(z-z^{(p)}\right)\right], \\
C_{i j l m}(z)=\sum_{p=1}^{6} C_{i j l m}^{(p)}(z)\left[\theta\left(z-z^{(p-1)}\right)-\theta\left(z-z^{(p)}\right)\right],
\end{gathered}
$$

where $\rho^{(p)}=\left\{\begin{array}{l}\rho_{w}, \\ \rho_{b},\end{array}\right.$ and $C_{i j l m}^{(p)}(z)=\left\{\begin{array}{l}C_{i j l m}^{(p)(w)}(z), \\ C_{i j l m}^{(p)(b)}(z),\end{array}\right.$, indices " $w$ " and " $b$ " denote the RTS layer corresponding to the potential well and the barrier, respectively.

If in relation (18) we go over to the Voigt notation with two indices and then take into account (20) and (21), equation (17) is equivalent to the following one:

$$
\begin{aligned}
& \rho(z) \frac{\partial^{2} \bar{u}}{\partial t^{2}}=\left(C_{12}+2 C_{44}\right) \nabla \cdot(\nabla \cdot \bar{u})-C_{44} \nabla \times(\nabla \times \bar{u}), \\
& \bar{u}=\bar{u}(x, y, z),
\end{aligned}
$$

Now, taking into account the plane geometry of the studied multilayer nanosystem, we can assume that

$$
\bar{u}(x, y, z)=\bar{u}(x, z)=\bar{u}(x) \bar{u}(z) .
$$

Moreover, the displacement vector $\bar{u}(z)$ in this case has two nonzero components

$$
\bar{u}(z)=\bar{u}_{x}(z)+\bar{u}_{z}(z)=\left(u_{x}(z) ; 0 ; u_{z}(z)\right) .
$$

Further, it is convenient to represent vectors $\bar{u}_{x}(z), \bar{u}_{z}(z)$ as the sum of two components:

$$
\begin{aligned}
& \bar{u}_{x}(z)=\bar{u}_{x}^{t}(z)+\bar{u}_{x}^{l}(z) ; \\
& \bar{u}_{x_{3}}(z)=\bar{u}_{z}^{t}(z)+\bar{u}_{z}^{l}(z),
\end{aligned}
$$

which satisfy the following conditions [10]:

$$
\begin{aligned}
& \left(\nabla \times \bar{u}_{x(z)}^{t}(z)\right)=0,\left(\nabla \cdot\left(\nabla \cdot \bar{u}_{x(z)}^{l}(z)\right)\right)=\nabla^{2} \bar{u}_{x(z)}^{l}(z) ; \\
& \left(\nabla \cdot \bar{u}_{x(z)}^{t}(z)\right)=0 ;\left(\nabla \times\left(\nabla \times \bar{u}_{x(z)}^{t}(z)\right)\right)=-\nabla^{2} \bar{u}_{x(z)}^{t}(z) .
\end{aligned}
$$

Now the solutions of equation (22), taking into account expression (23) with conditions (25) and (26), should be sought in the form [10]:

$$
\bar{u}(x, y, z)=\bar{u}(x, z)=\bar{u}(z) e^{i(q x-\omega t)} .
$$

In this case, equation (22) for an arbitrarily selected $p$-th layer of the nanostructure is equivalent to the following two equations:

$$
-\frac{d^{2} u_{x}^{l(p)}(z)}{d z^{2}}+\left(q^{2}-\frac{\omega^{2}}{v_{l_{p}}^{2}}\right) u_{x_{1}}^{l(p)}\left(x_{3}\right)=0 ;-\frac{d^{2} u_{x}^{t(p)}(z)}{d z^{2}}+\left(q^{2}-\frac{\omega^{2}}{v_{t_{p}}^{2}}\right) u_{x}^{t(p)}\left(x_{3}\right)=0
$$

where propagation velocities of the longitudinal and transverse waves in a separate nanosystem layer

$$
v_{l}^{(p)}=\sqrt{\frac{C_{12}^{(p)}+2 C_{44}^{(p)}}{\rho^{(p)}}} ; v_{t}^{(p)}=\sqrt{\frac{C_{44}^{(p)}}{\rho^{(p)}}},
$$

$\omega$ is the cyclic frequency, $q$ is the wave vector.

The solutions of equations (28) have the form:

$$
\begin{aligned}
& u_{x}^{l(p)}(z)=A_{l}^{(p)} e^{-\chi_{l}^{(p)}\left(z-z_{p-1}\right)}+B_{l}^{(p)} e^{\chi_{l}^{(p)}\left(z-z_{p-1}\right)} ; \\
& u_{z}^{t(p)}(z)=A_{t}^{(p)} e^{-\chi_{t}^{(p)}\left(z-z_{p-1}\right)}+B_{t}^{(p)} e^{\chi_{t}^{(p)}\left(z-z_{p-1}\right)} ; \\
& \chi_{l}^{(p)}=\sqrt{q^{2}-\frac{\omega^{2}}{v_{l_{p}}^{2}}} ; \chi_{t}^{(p)}=\sqrt{q^{2}-\frac{\omega^{2}}{v_{t_{p}}^{2}}} .
\end{aligned}
$$

The components $u_{x_{3}}^{l(p)}\left(x_{3}\right)$ and $u_{x_{3}}^{t(p)}\left(x_{3}\right)$ are obtained using conditions (26). Hence we have:

$$
\frac{\partial \bar{u}_{x}^{l}(z)}{\partial z}-\frac{\partial \bar{u}_{z}^{l}(z)}{\partial x}=0 ; \frac{\partial \bar{u}_{x}^{t}(z)}{\partial x}+\frac{\partial \bar{u}_{z}^{t}(z)}{\partial z}=0 .
$$

Then taking into account (27) up to a constant we have:

$$
\begin{gathered}
u_{z}^{l}(z)=-\frac{i}{q} \frac{\partial u_{x}^{l}(z)}{\partial z}=i \frac{\chi_{l}^{(p)}}{q}\left(A_{l}^{(p)} e^{-\chi_{l}^{(p)}\left(z-z_{p}\right)}-B_{l}^{(p)} e^{\chi_{l}^{(p)}\left(z-z_{p}\right)}\right) \\
i q u_{x}^{t}(z)+\frac{\partial u_{z}^{t}(z)}{\partial z}=0 \\
u_{z}^{t}(z)=i \frac{q}{\chi_{t}^{(p)}}\left(A_{t}^{(p)} e^{-\chi_{t}^{(p)}\left(z-z_{p}\right)}-B_{t}^{(p)} e^{\chi_{t}^{(p)}\left(z-z_{p}\right)}\right)
\end{gathered}
$$

Thus, the components of the displacement field for the studied nanosystem can generally be represented in following form: 


$$
\begin{aligned}
& u_{x}(z)=u_{x}^{l(p)}(z)+u_{x}^{t(p)}(z)=\left(B_{l}^{(0)} e^{\chi_{l}^{(0)} z}+B_{t}^{(0)} e^{\chi_{t}^{(0)}} z\right) \theta(-z)+\left(A_{l}^{(6)} e^{-\chi_{l}^{(b)}\left(z-z_{5}\right)}+A_{t}^{(6)} e^{-\chi_{t}^{(p)}\left(z-z_{5}\right)}\right) \theta\left(z-z_{5}\right)+ \\
& +\sum_{p=1}^{5}\left(A_{l}^{(p)} e^{-\chi_{l}^{(p)}\left(z-z_{p}\right)}+B_{l}^{(p)} e^{\chi_{l}^{(p)}\left(z-z_{p}\right)}+A_{t}^{(p)} e^{-\chi_{t}^{(p)}\left(z-z_{p}\right)}+B_{t}^{(p)} e^{\chi_{t}^{(p)}\left(z-z_{p}\right)}\right)\left[\theta\left(z-z_{p-1}\right)-\theta\left(z-z_{p}\right)\right] ; \\
& u_{z}(z)=u_{z}^{t}(z)+u_{z}^{l}(z)=-i\left(\frac{\chi_{l}^{(p)}}{q} B_{l}^{(p)} e^{\chi_{l}^{(p)} z}+\frac{q}{\chi_{t}^{(p)}} B_{t}^{(p)} e^{\chi_{t}^{(p)} z}\right) \theta(-z)+ \\
& +i\left(\frac{\chi_{l}^{(p)}}{q}\left(A_{l}^{(p)} e^{-\chi_{l}^{(p)}\left(z-z_{p}\right)}-B_{l}^{(p)} e^{\chi_{l}^{(p)}\left(z-z_{p}\right)}\right)+\frac{q}{\chi_{t}^{(p)}}\left(A_{t}^{(p)} e^{-\chi_{l}^{(p)}\left(z-z_{p}\right)}-B_{t}^{(p)} e^{\chi_{l}^{(p)}\left(z-z_{p}\right)}\right)\right)\left[\theta\left(z-z_{p-1}\right)-\theta\left(z-z_{p}\right)\right]+ \\
& +i \sum_{p=1}^{5}\left(\frac{\chi_{l}^{(p)}}{q} A_{l}^{(6)} e^{-\chi_{l}^{(p)}\left(z-z_{5}\right)}+\frac{q}{\chi_{t}^{(p)}} A_{t}^{(6)} e^{-\chi_{t}^{(p)}\left(z-z_{5}\right)}\right)\left[\theta\left(z-z_{p-1}\right)-\theta\left(z-z_{p}\right)\right] .
\end{aligned}
$$

In relations (34), it is taken into account that $A_{l}^{(0)}=A_{t}^{(0)}=0 ; B_{l}^{(5)}=B_{t}^{(5)}=0$, since in the external environment, in which the RTS is located (at $z<0$ and $z>z_{5}$ ), the components of the elastic displacement must decrease to zero at $x_{3} \rightarrow \pm \infty$, which is fully ensured by the fulfillment of the condition:

$$
\left.u_{x_{1(3)}}\left(x_{3}\right)\right|_{x_{3} \rightarrow \pm \infty} \rightarrow 0 .
$$

\section{Unknown}

coefficients $B_{l}^{(0)}, B_{t}^{(0)}, A_{l}^{(6)}, A_{t}^{(6)}, A_{l}^{(p)}, B_{l}^{(p)}, A_{t}^{(p)}, B_{t}^{(p)}$ in the solutions (34) are found using the boundary conditions for the components of the displacement vector $u_{x}(z), u_{z}(z)$ and stress tensor $\sigma_{z x}, \sigma_{z z}$ at all heteroboundaries of the investigated RTS:

$$
\begin{aligned}
& \left\{\begin{array}{l}
\left.u_{x(z)}^{(p)}(z)\right|_{z \rightarrow z^{(p)}-\varepsilon}=\left.u_{x(z)}^{(p+1)}(z)\right|_{z \rightarrow z^{(p)}+\varepsilon} \\
\left.\sigma_{z x}^{(p)}(z)\right|_{z \rightarrow z^{(p)}-\varepsilon}=\left.\sigma_{z x}^{(p+1)}(z)\right|_{z \rightarrow z^{(p)}+\varepsilon} \\
\left.\sigma_{z z}^{(p)}(z)\right|_{z \rightarrow z^{(p)}-\varepsilon}=\left.\sigma_{z z}^{(p)}(z)\right|_{z \rightarrow z^{(p)}+\varepsilon}
\end{array}\right. \\
& \varepsilon \rightarrow 0 ; p=0-5 .
\end{aligned}
$$

where the components of the stress tensor are defined as [10]:

$$
\begin{aligned}
& \sigma_{z x}^{(p)}\left(x_{3}\right)=C_{44}^{(p)}\left(\frac{\partial \bar{u}_{z}^{(p)}(z)}{\partial x}+\frac{\partial \bar{u}_{z}^{(p)}(z)}{\partial z}\right)=C_{44}^{(p)}\left(i q u_{z}^{(p)}(z)+\frac{\partial u_{x_{1}}^{(p)}(z)}{\partial z}\right) e^{i q x}= \\
& =C_{44}^{(p)}\left(-2 \chi_{l}^{(p)} A_{l}^{(p)} e^{-\chi_{l}^{(p)}\left(z-z_{p}\right)}+2 \chi_{l}^{(p)} B_{l}^{(p)} e^{\chi_{l}^{(p)}\left(z-z_{p}\right)}-\left(\frac{q^{2}}{\chi_{t}^{(p)}}+\chi_{t}^{(p)}\right) A_{t}^{(p)} e^{-\chi_{t}^{(p)}\left(z-z_{p}\right)}+\left(\frac{q^{2}}{\chi_{t}^{(p)}}+\chi_{t}^{(p)}\right) B_{t}^{(p)} e^{\chi_{t}^{(p)}\left(z-z_{p}\right)}\right) e^{i q x} \\
& \sigma_{z z}^{(p)}\left(x_{3}\right)=C_{12}^{(p)} \nabla \bar{u}(x, z)+2 C_{44}^{(p)} \frac{\partial \bar{u}_{z}^{(p)}(z)}{\partial z}=\left(i q C_{12}^{(p)} u_{x}^{(p)}(z)+\left(C_{12}^{(p)}+2 C_{44}^{(p)}\right) \frac{\partial u_{z}^{(p)}(z)}{\partial z}\right) e^{i q x}= \\
& =\left(i q C_{12}^{(p)} u_{x}^{(p)}(z)+C_{11}^{(p)} \frac{\partial u_{z}^{(p)}(z)}{\partial z}\right) e^{i q x}=i\left(\left(q C_{12}^{(p)}-\frac{\left(\chi_{l}^{(p)}\right)^{2}}{q} C_{11}^{(p)}\right) A_{l}^{(p)} e^{-\chi_{l}^{(p)}\left(z-z_{p}\right)}+\right. \\
& \left.+\left(q C_{12}^{(p)}-\frac{\left(\chi_{l}^{(p)}\right)^{2}}{q} C_{11}^{(p)}\right) B_{l}^{(p)} e^{\chi_{l}^{(p)}\left(z-z_{p}\right)}+q\left(C_{12}^{(p)}-C_{11}^{(p)}\right) A_{t}^{(p)} e^{-\chi_{t}^{(p)}\left(z-z_{p}\right)}+q\left(C_{12}^{(p)}-C_{11}^{(p)}\right) B_{t}^{(p)} e^{\chi_{t}^{(p)}\left(z-z_{p}\right)}\right) \\
& C_{12}^{(p)}=C_{11}^{(p)}-2 C_{44}^{(p)} .
\end{aligned}
$$

The dispersion equation for finding the spectrum of acoustic phonons $\Omega=\Omega(q)$ is obtained by determining the ratio between the coefficients $A_{l}^{(p)}, B_{l}^{(p)}, A_{t}^{(p)}, B_{t}^{(p)}$

of $p$-th and $(p+1)$-th RTS layers using transfer-matrix method [10-12]:

$$
\left(\begin{array}{llll}
A_{l}^{(p)} & B_{l}^{(p)} & A_{t}^{(p)} & B_{t}^{(p)}
\end{array}\right)^{T}=T^{(p, p+1)}(q)\left(A_{l}^{(p+1)} \quad B_{l}^{(p+1)} \quad A_{t}^{(p+1)} \quad B_{t}^{(p+1)}\right)^{T} ; T^{(p, p+1)}(q)=M_{p}^{-1}(q) M_{p+1}(q),
$$

where, in accordance with the boundary conditions (36), the matrix $M_{p}(q)$ is defined as

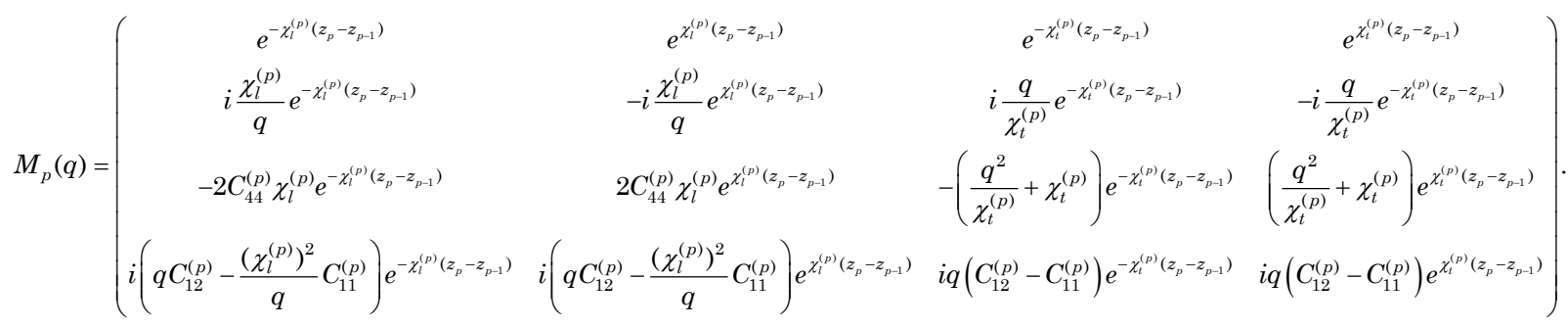


Next, it is necessary to express the coefficients $B_{l}^{(0)}, B_{t}^{(0)}$ of the solutions for the medium at $z<0$ in terms of the coefficients $A_{l}^{(6)}, A_{t}^{(6)}$ of the solutions for the medium at $z>z_{5}$ :

$$
\left(\begin{array}{llll}
0 & B_{l}^{(0)} & 0 & B_{t}^{(0)}
\end{array}\right)^{T}=T^{(0,6)}(q, \omega)\left(A_{l}^{(6)} \quad 0 \quad A_{t}^{(6)} \quad 0\right)^{T},(40)
$$

where the transfer matrix of the nanosystem

$$
T^{(0,6)}=T(q, \omega)=\prod_{p=0}^{5} T^{(p, p+1)}(q, \omega) .
$$

The dispersion equation that describes the dependences of the spectrum of acoustic phonons $\Omega_{n_{1} q}(q)=\hbar \omega_{n_{1}, q}(q)$ is obtained as

$$
|T(q, \omega)|=\left|\prod_{p=0}^{5} T^{(p, p+1)}(q, \omega)\right|=0 .
$$

Using the boundary conditions (36), all the coefficients in the solutions (34) are expressed through one of them, in our case it is a coefficient $B_{l}^{(0)}$. This coefficient is obtained from the normalization condition

$$
\left|B_{l}^{(0)}\right|^{2} \int_{-\infty}^{\infty} \rho(z)\left(\left|u_{x}(z)\right|^{2}+\left|u_{z}(z)\right|^{2}\right) d z=\frac{\hbar}{2 \omega_{\lambda, q} l_{x} l_{y}} .
$$

Thus, we obtain the Hamiltonian of acoustic phonons in terms of the occupation numbers $[5,8]$ :

$$
\hat{H}_{\mathrm{ac}}(q)=\sum_{n_{1}} \Omega_{n}(q)\left[b_{n_{1}}^{+}(q) b_{n_{1}}(q)+\frac{1}{2}\right],
$$

where $b_{n}^{+}(q)$ and $b_{n}(q)$ are respectively the bosonic acoustic phonon states creation and annihilation operators.

\section{HAMILTONIAN OF ELECTRON-ACOUSTIC PHONON INTERACTION. SHIFTS OF THE ELECTRONIC SPECTRUM AND DECAY RATES OF THE ELECTRONIC LEVELS}

Taking into account the normalization condition (43), the elastic displacement of the nanosystem medium acquires the following form:

$$
\begin{aligned}
& \hat{u}(q, \omega, r)=\sum_{p=1}^{5} \sum_{q, n_{1}} \sqrt{\frac{\hbar}{2 l_{x} l_{y} \omega_{n_{1}}}}\left(b_{n_{1}}(q)+b_{n_{1}}^{+}(-q)\right) \times \\
& \times\left(\begin{array}{l}
u_{x n_{1}}^{(p)}\left(\omega_{n_{1}}, z\right) \\
u_{z n_{1}}^{(p)}\left(\omega_{n_{1}}, z\right)
\end{array}\right) e^{-i q r}\left[\theta\left(z-z_{p-1}\right)-\theta\left(z-z_{p+1}\right)\right] .
\end{aligned}
$$

For GaAlAs arsenide semiconductors, the deformation potential is expressed as

$$
H_{d e f}=H_{0}(\nabla \cdot \bar{u}),
$$

where $H_{0}$ is the deformation potential constant, which, taking into account (45), gives the expression for the interaction Hamiltonian through the deformation potential in the second quantization representation:

$$
\begin{aligned}
& \hat{H}_{d e f}=\sum_{p=1}^{5} \sum_{q, n_{1}} \sqrt{\frac{\hbar H_{0}^{2}}{2 l_{x} l_{y} \omega_{n_{1}}}}\left(b_{n_{1}}(q)+b_{n_{1}}^{+}(-q)\right)\left(-i q u_{x n_{1}}^{(p)}\left(\omega_{n_{1}}, z\right)+\frac{\partial u_{z n_{1}}^{(p)}\left(\omega_{n_{1}}, z\right)}{\partial z}\right) e^{-i q r}\left[\theta\left(z-z_{p-1}\right)-\theta\left(z-z_{p+1}\right)\right]= \\
& =-\sum_{p=1}^{5} \sum_{q, n_{1}} i \sqrt{\frac{\hbar H_{0}^{2}}{2 l_{x} l_{y} \omega_{n_{1}}}}\left(b_{n_{1}}(q)+b_{n_{1}}^{+}(-q)\right)\left\{\left(q+\frac{\left(\chi_{l}^{(p)}\right)^{2}}{q}\right)\left(A_{l}^{(p)} e^{-\chi_{l}^{(p)}\left(z-z_{p}\right)}+B_{l}^{(p)} e^{\chi_{l}^{(p)}\left(z-z_{p}\right)}\right)+\right. \\
& \left.+2 q\left(A_{t}^{(p)} e^{-\chi_{t}^{(p)}\left(z-z_{p}\right)}+B_{t}^{(p)} e^{\chi_{t}^{(p)}\left(z-z_{p}\right)}\right)\right\} e^{-i q r}\left[\theta\left(z-z_{p-1}\right)-\theta\left(z-z_{p+1}\right)\right] .
\end{aligned}
$$

Then, the Hamiltonian, which describes the interaction of an electron with acoustic phonons, takes the following form:

$$
\begin{gathered}
\hat{H}_{e-a c . p h}=\sum_{n, n^{\prime}, n_{1}, \bar{k}, \bar{q}} F_{n n_{1}}(q) \hat{a}_{n^{\prime}, \bar{k}+\bar{q}}^{+} \widehat{a}_{n \bar{k}}\left[b_{n_{1}}(q)+b_{n_{1}}^{+}(-q)\right],(48) \\
F_{n n_{1}}(q)=-\sqrt{\frac{\hbar H_{0}^{2}}{2 l_{x} l_{y} \rho^{(p)} \omega_{n_{1}}}} \times \int_{z_{p-1}}^{z_{p}} \Psi_{E \bar{k}}\left(E_{n}, z\right)\left\{\left(q+\frac{\left(\chi_{l}^{(p)}\right)^{2}}{q}\right)\left(A_{l}^{(p)} e^{-\chi_{l}^{(p)}\left(z-z_{p}\right)}+B_{l}^{(p)} e^{\chi_{l}^{(p)}\left(z-z_{p}\right)}\right)+\right. \\
\left.+2 q\left(A_{t}^{(p)} e^{-\chi_{t}^{(p)}\left(z-z_{p}\right)}+B_{t}^{(p)} e^{\chi_{t}^{(p)}\left(z-z_{p}\right)}\right)\right\} \Psi_{E \bar{k}}^{*}\left(E_{n}, z\right) d z .
\end{gathered}
$$

sions (10) and (47) are defined as:
Thus, the Hamiltonian of the electron-acoustic phonon system:

$$
\begin{aligned}
& \hat{H}=\hat{H}_{e}+\hat{H}_{a c}+\hat{H}_{e-a c \cdot p h}= \\
& =\sum_{n \bar{k}} E_{n \bar{k}} \hat{a}_{n \bar{k}}^{+} \hat{a}_{n \bar{k}}+\sum_{n_{1}} \Omega_{n}(q)\left[b_{n_{1}}^{+}(q) b_{n_{1}}(q)+\frac{1}{2}\right]+ \\
& +\sum_{n, n^{\prime}, n_{1}, \bar{k}, \bar{q}} F_{n n_{1}}(q) \hat{a}_{n^{\prime}, \bar{k}+\bar{q}}^{+} \hat{a}_{n \bar{k}}\left[b_{n_{1}}(q)+b_{n_{1}}^{+}(-q)\right] .
\end{aligned}
$$

For a discrete electronic spectrum, its renormalization by interaction with acoustic phonons is obtained by performing the Fourier transform of the Green's function, which is determined from the Dyson equation:

$$
G_{n}(\Omega)=\left(\Omega-E_{n}-M_{n}(\Omega)\right)^{-1},
$$

where the mass operator is 


$$
\begin{aligned}
& M_{n}(\Omega, \bar{k})=\sum_{q n_{1} n^{\prime}}\left|F_{n n_{1} n^{\prime}}(q)\right|^{2}\left\{\frac{1+v_{n_{1}}}{\Omega-E_{n^{\prime}, \bar{k}+\bar{q}}-\hbar \omega_{n_{1}}+i \eta}+\right. \\
& \left.+\frac{v_{n_{1}}}{\Omega-E_{n^{\prime}, \bar{k}+\bar{q}}-\hbar \omega_{n_{1}}+i \eta}\right\} ; \eta \rightarrow 0,
\end{aligned}
$$

and $v_{n_{1}}=\left(e^{h \omega_{n_{1}} / k T}-1\right)^{-1}$ is the occupation number of the acoustic phonon states.

Next, we will consider the electron motion perpendicular to the layers of the nanostructure. Then, assuming that $\bar{k}=0$, we find the renormalized electronic spectrum from the dispersion equation, which in turn follows from the relations (51) and (52):

$$
\hbar \omega-\tilde{E}_{n}-M_{n}(\Omega)=0 .
$$

Whence the shifts of the electronic states and their decay rates are obtained as:

$$
\begin{aligned}
& \Delta_{n}=\underset{\Omega=E_{n}, \bar{k}=0}{\operatorname{Re}} M_{n}(\Omega)=\frac{l_{x} l_{y}}{(2 \pi)^{2}} \sum_{n_{1}} \mathrm{P} \iint \frac{\left|F_{n n_{1} n}(q)\right|^{2} d q^{2}}{E_{n}-E_{n, \bar{q}}-\hbar \omega_{n_{1}}} ; \\
& \Gamma_{n}=-2 \operatorname{Im}_{\Omega=E_{n}, \bar{k}=0} M_{n}(\Omega)=\frac{l_{x} l_{y}}{2 \pi} \sum_{n_{1}} \iint \frac{\left|F_{n n_{1} n}(q)\right|^{2} d q^{2}}{\delta\left(E_{n}-E_{n, \bar{q}}-\hbar \omega_{n_{1}}\right)} .
\end{aligned}
$$

where the symbol "P" means that the integral must be taken via the Cauchy principal value.

Then, the stationary levels of the electronic spectrum renormalized by the interaction with acoustic phonons are defined as

$$
\tilde{E}_{n}=E_{n}+\Delta_{n} .
$$

\section{DISCUSSION OF THE RESULTS}

The calculations of the electronic spectrum of acoustic phonons, shifts of the stationary electronic spectrum levels and their decay rates were carried out on the basis of the theory developed above. The geometric parameters of a double-well nanostructure used in direct calculations are the following: quantum well widths $d_{1}=d_{2}=10 \mathrm{~nm}$, thicknesses of potential barriers $\Delta_{1}=\Delta_{2}=\Delta_{3}=2 \mathrm{~nm}$. The geometrical parameters of the nanostructure cross-sectional area are taken as $l_{x}=l_{y}=10^{-6} \mathrm{~m}$. The value of the potential barrier is $U_{0}=520 \mathrm{meV}$. The deformation potential constants are as follows: $H_{0}^{\mathrm{GaAs}}=-7.17 \mathrm{eV}, H_{0}^{\mathrm{AlAs}}=-5.64 \mathrm{eV}$. Other physical parameters of $\mathrm{GaAs}$ and $\mathrm{AlAs}$ semiconductors are given in Table 1.

Table 1 - Physical parameters of GaAs and AlAs semiconductors

\begin{tabular}{|c|c|c|c|c|c|}
\hline & $m / m_{\mathrm{e}}$ & $\begin{array}{c}P \\
\left(\mathrm{~kg} / \mathrm{m}^{3}\right)\end{array}$ & $\begin{array}{c}C_{11} \\
(\mathrm{GPa})\end{array}$ & $\begin{array}{c}C_{12} \\
(\mathrm{GPa})\end{array}$ & $\begin{array}{c}C_{44} \\
(\mathrm{GPa})\end{array}$ \\
\hline $\mathrm{GaAs}$ & 0.063 & 5320 & 106.5 & 60.2 & 33.6 \\
\hline $\mathrm{AlAs}$ & 0.146 & 3760 & 119.9 & 57.5 & 56.6 \\
\hline
\end{tabular}

The dependences of the first four stationary electronic spectrum levels $E_{n}$ on the value of $d$ $\left(0 \leq d \leq d_{1}+d_{2}\right)$, i.e., on the position of the internal potential barrier in the total potential well, are shown in Fig. 2.

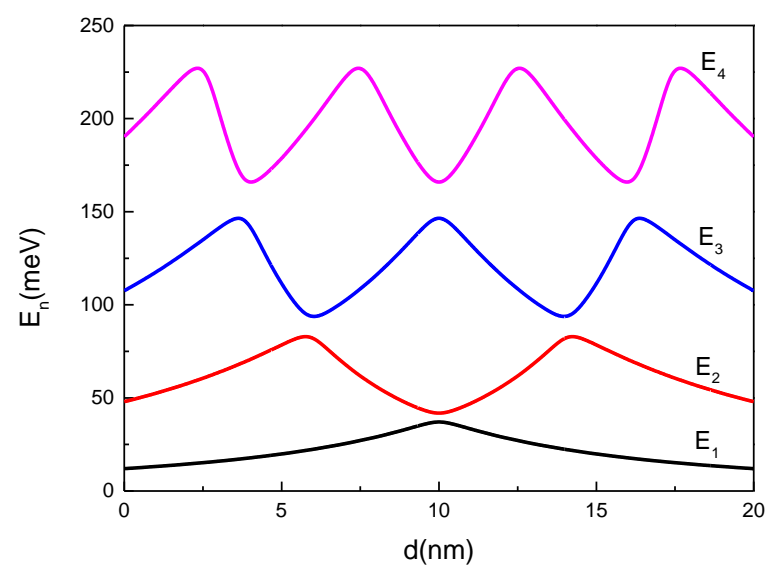

Fig. 2 - Dependences of the stationary electronic spectrum $E_{n}$ on $d=d_{1}+d_{2}$

As can be seen from Fig. 2, the dependences of the electron energy spectrum form with increasing $d$, respectively, of $(n-1)$ minima and $n$ maxima, which corresponds to the level number $n$. In addition, the dependences $E_{n}(d)$ are symmetric about the middle of the total potential well, i.e., the point $\tilde{d}=\left(d_{1}+d_{2}\right) / 2$.

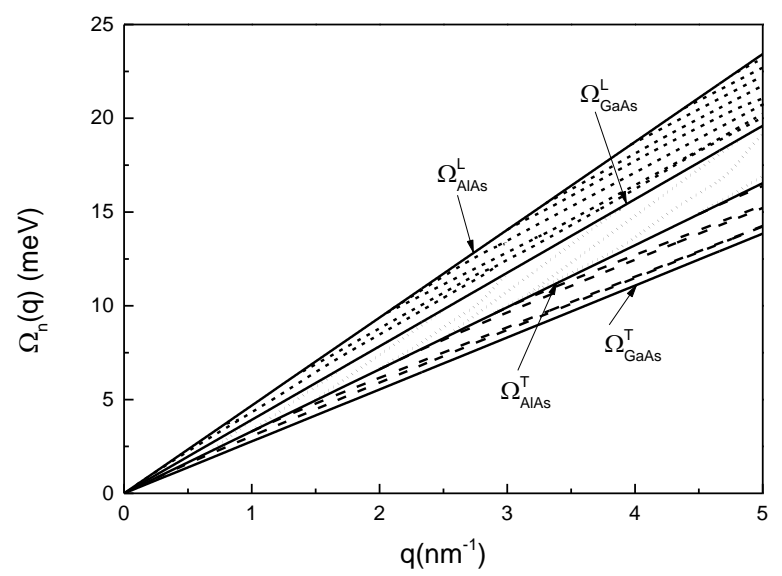

Fig. 3 -Dependences of the stationary electronic spectrum $E_{n}$ on $d=d_{1}+d_{2}$

Further, in Fig. 3, the spectral dependences for acoustic phonons as a function of the wave vector $q$ are shown. As seen from this figure, the dependences are formed by a set of branches located within three regions, which are determined in the order of increasing energy values $\Omega_{\mathrm{GaAs}}^{\mathrm{T}}, \Omega_{\mathrm{AlAs}}^{\mathrm{T}}, \Omega_{\mathrm{GaAs}}^{\mathrm{L}}, \Omega_{\mathrm{AlAs}}^{\mathrm{L}}$, in accordance with certain group propagation velocities of the transverse (index "T") and longitudinal (index "L") waves in GaAs and AlAs semiconductor materials. Dependences $\Omega_{n_{1}}(q)$ within these regions are formed, respectively, at the values: for the first group - at $\Omega_{\mathrm{AlAs}}^{\mathrm{T}}$, for the second - similarly at $\Omega_{\mathrm{AlAs}}^{\mathrm{T}}$, and for the third - at $\Omega_{\text {AlAs }}^{\mathrm{L}}$. With an increase in the wave vector values $q$, the energies of acoustic phonons also increase quasilinearly within the given 
three regions. It should be noted that the dependences in the first $\left(\Omega_{\mathrm{GaAs}}^{\mathrm{T}} \leq \Omega_{n_{1}}(q) \leq \Omega_{\mathrm{AlAs}}^{\mathrm{T}}\right.$ ) and the third $\left(\Omega_{\mathrm{GaAs}}^{\mathrm{L}} \leq \Omega_{n_{1}}(q) \leq \Omega_{\mathrm{AlAs}}^{\mathrm{L}}\right)$ regions have similar behavior, but in the first region, phonon branches form in pairs, and in the third region, the branches approach each other with increasing $q$. The dependency branches formed within the second group $\left(\Omega_{\text {AlAs }}^{\mathrm{T}} \leq \Omega_{n_{1}}(q) \leq \Omega_{\text {GaAs }}^{\mathrm{L}}\right.$ ) radically differ from the other two groups due to the different sign of their dispersion from $q$. In addition, in this group of depend-
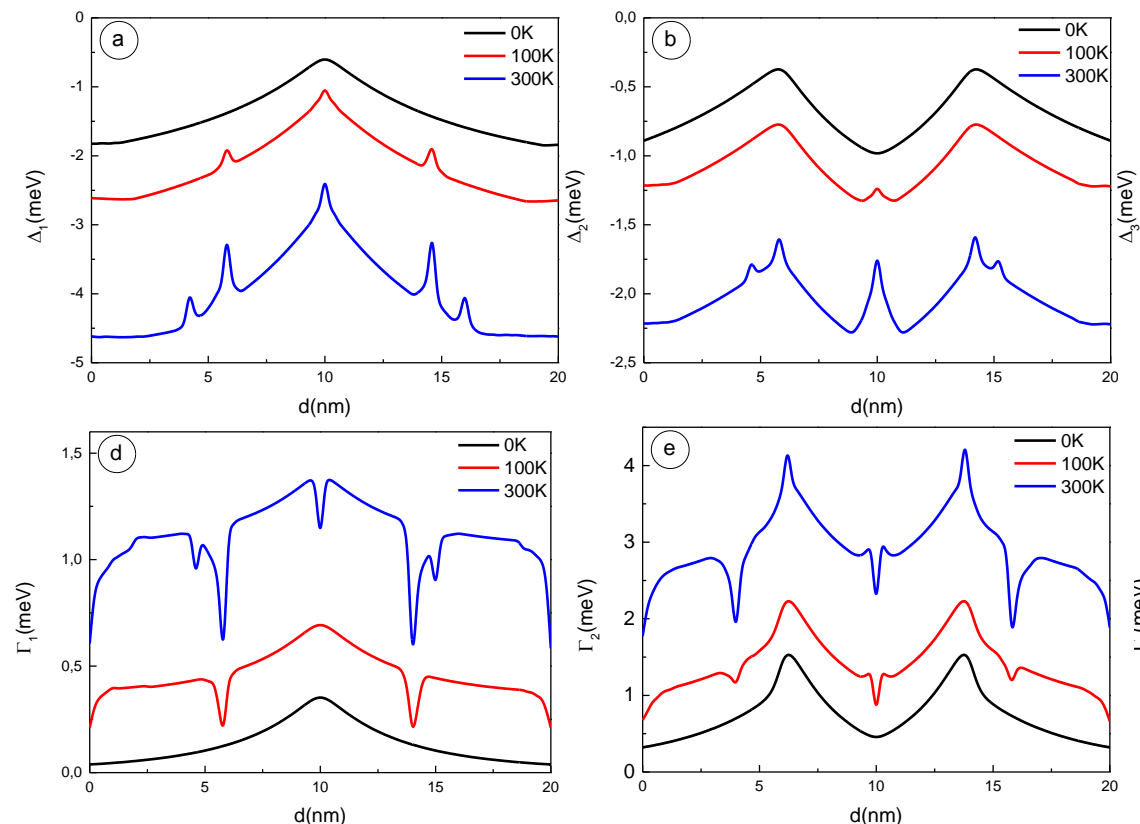

ency branches there are those that have a weak quasiquadratic dependence on $q$.

Further, in Fig. 4, the dependences of the stationary electronic spectrum level shifts and their decay rates are presented, which were calculated at three different temperatures: $0 \mathrm{~K}, 100 \mathrm{~K}$ and $300 \mathrm{~K}$. These temperature values are relevant for the next reasons: the simplest case of electron-phonon interaction (0 K), cooling of QCL and QCD by liquid nitrogen (operating temperature range of about 80-100 K), QCL and QCD operating at room temperature (300 K).

Fig. 4 - Dependences of temperature shifts $(\mathrm{a}, \mathrm{b}, \mathrm{c})$ for the electronic spectrum levels $(n=1,2,3)$ and their decay rates $(\mathrm{d}$, e, f) on $d=d_{1}+d_{2}$ at different temperature values

As can be seen from Fig. 4a, b, c, the dependences of temperature shifts on $d$ are similar to the behavior of the levels of the stationary electronic spectrum, being also symmetric relative to the center of the total potential well. This is quite true for the dependences calculated at $0 \mathrm{~K}$. However, with an increase in temperature $(100 \mathrm{~K})$, despite the symmetry of the dependences being maintained, additional maxima are formed associated with the contribution of the remaining electronic levels to the spectrum renormalization. At room temperature, we have a significant increase in the maxima formed at $100 \mathrm{~K}$, and two additional maxima are also formed. In general, it should be concluded that with increasing temperature, the shifts of the electronic spectrum increase, with lower energy levels shifting more, which leads to a decrease in the frequency of the electronic transitions in the nanostructure.

Further, in Fig. 4d, e, f, the dependences on $d$ for the decay rates $\Gamma_{n}(d)$ of the electronic spectrum levels in the investigated RTS are shown. These dependences are also symmetric relative to the center of the total potential well, as well as the dependences of temperature shifts. It should be noted that in most cases, with increasing temperature, the maxima of the dependences that are formed in $\Gamma_{n}(d)$ correspond to the minima of the dependences $\Delta_{n}(d)$. This behavior of the electronic states decay rates is determined by the behavior of the functions $\delta\left(E_{n}-E_{n, \bar{q}}-\hbar \omega_{n_{1}}\right)$ and the dependence of these quantities on temperature through the $v_{n_{1}}(T)$ function. Thus, due to the electron-acoustic phonon interaction, in addition to renormalizing the energies of quantum transitions, the absorption bands also change.

\section{CONCLUSIONS}

A quantum-mechanical theory of the interaction of electrons with acoustic phonons in an arsenidebased AlAs/GaAs resonant tunneling nanostructure is developed. Based on the developed theory, the temperature shifts of stationary electronic levels and their decay rates depending on the position of the internal potential barrier in the total potential well were calculated. The results of these calculations, performed for different temperatures, show that an increase in temperature causes an increase in the shifts of the electronic spectrum levels and their decay rates. In practice, this affects the need to adjust the calculated operating frequency of the working QCLs and QCDs. 


\section{REFERENCES}

1. Q.Y. Lu, S. Manna, D.H. Wu, S. Slivken, M. Razeghi, Appl. Phys. Lett. 112, 14 (2018).

2. M.P. Semtsiv, W.T. Masselink, Appl. Phys. Lett. 109, 20 (2016).

3. L. Li, D.Y. Xiong, Z. Tang, J. Wen, N. Li, P.P. Chen, Z.Q. Zhu, J. Appl. Phys. 121, 8 (2017).

4. L. Bosco, M. Franckié, G. Scalari, M. Beck, A. Wacker, J. Faist, Appl. Phys. Lett. 115, 1 (2019).

5. M.V. Tkach, Ju.O. Seti, Y.B. Grynyshyn, O.M. Voitsekhivska, Condens. Matter Phys. 7, 2 (2014).

6. J.-J. Shi, B.C. Sanders, S.-H. Pan, Eur. Phys. J. B 8, 4 (1998).
7. S.M. Komirenko, K.W. Kim, A.A. Demidenko, V.A. Kochelap, M.A. Stroscio, J. Appl. Phys. 90, 8 (2001).

8. S.M. Komirenko, K.W. Kim, V.A. Kochelap, M.A. Stroscio, Phys. Rev. B 65, 15 (2002).

9. B. Krummheuer, V.M. Axt, T. Kuhn, Phys. Rev. B 72, 24 (2005).

10. I. Boyko, M. Petryk, J. Nano- Electron. Phys. 11 No 1, 01019 (2019).

11. I. Boyko, M. Petryk, J. Fraissard, Eur. Phys. J. B 93, 57 (2020)

12. J. Piprek, Semiconductor Optoelectronic Devices (Elsevier Inc.: 2003).

\title{
Взаемодія електронів з акустичними фононами у AlAs/GaAlAs резонансно-тунельних наноструктурах
}

\author{
I.В. Бойко, М.Р. Петрик
}

Тернопільський національний технічний університет ілені Івана Пулюя, вул. Руська, 56, 46001 Тернопіль, Україна

\begin{abstract}
У статті, з використанням точних розв'язків стаціонарного рівняння Шредінгера та рівняння руху пружного напівпровідникового середовища, на базі формалізму вторинного квантування, розвинута теорія взаемодії електронів з акустичними фононами в арсенідній багатошаровій резонансній тунельній структурі AlAs/GaAlAs. 3 використанням мацубарівських функцій Гріна та рівняння Дайсона встановлені вирази, які описують температурні зміщення енергій електронних рівнів в наноструктурі і швидкості їх згасання. Безпосередні розрахунки величин, що характеризують взаемодію електронів з акустичними фононами, виконано на основі фізичних і геометричних параметрів типової наноструктури. Послідовно досліджено залежності перенормованих спектральних параметрів електрона від геометричних параметрів сумарної потенціальної ями наносистеми при різних температурах. Показано, що вплив акустичних фононів спричиняе зменшення частоти квантових електронних переходів у досліджуваній наноструктурі, а цей ефект стае більш помітним з ростом температури. Встановлено, що абсолютні величини температурних зміщень електронних стаціонарних станів зменшуються зі збільшенням номера електронного стаціонарного рівня. Також ріст температури спричиняе ріст часів розсіювання електронних станів, що є ефектом дисипації, безпосередньо впливаючи на електронні процеси в наноструктурі.
\end{abstract}

Ключові слова: Акустичний фонон, Електрон-фононна взаемодія, Рівняння Дайсона, Зміщення електронного стану, Згасання енергетичного стану. 